\title{
Distraction Osteogenesis
}

\author{
Hossein Behnia, Azita Tehranchi and Golnaz Morad \\ Additional information is available at the end of the chapter \\ http://dx.doi.org/10.5772/54647
}

\section{Introduction}

Distraction osteogenesis (DO) is defined as the formation of new bone between the vascular surfaces of osteotomized bone segments, separated gradually by distraction forces. [1] The incipient concept of distraction osteogenesis, as first described for correction of limb length discrepancies by Codivilla [2] in 1905, represented an osteotomized femur subjected to repeated forces of traction and counter-traction. This technique achieved a length increase of 3 to $8 \mathrm{~cm}$; an amount that surpassed that attainable by other methods common at that time. Codivilla asserted that confronting the resistance of the muscles surrounding a bone is inevitable if the discrepancies are to be corrected. Abbott and Saunders later used the technique for elongation of tibia. [3] Distraction osteogenesis proved advantageous over other conventional methods for management of bone defects, particularly bone grafting, in that it provided simultaneous expansion of the functional soft tissue matrix, referred to as distraction histogenesis. [1] The method however, remained undeveloped until it resurged in 1950s by Ilizarov, leading to several successful endeavors increasing the length of the extremities. In 1992, McCarthy [4] expanded the application of distraction osteogenesis to the craniofacial skeleton by attempting to ameliorate mandibular length deficiency in patients with hemifacial microsomia and Nager's syndrome. Accomplishing an average increase of $20 \mathrm{~mm}$ in the mandibular length in these preliminary cases, craniofacial DO rendered promising insights for treatment of craniofacial skeletal abnormalities. Hitherto, a plethora of treatment protocols and modalities have evolved in order to improve the outcomes of craniofacial DO.

\section{The biology of distraction osteogenesis}

Distraction osteogenesis initiates by surgically simulating bone fractures via osteotomy of the deficient bone. Normal fracture healing occurs through a cascade of molecular and cellular 
events triggered in response to injury. Formation of hematoma followed by chondrogenesis and angiogenesis eventually leads to the formation of hard callus by means of intramembranous and endochondral ossification. The resultant woven bone subsequently remodels into a more mature lamellar bone to restore the strength and function of the organ. [5], [6] During distraction osteogenesis however, the application of mechanical forces to the bone segments alters the repair process of the osteotomized bone segments, characteristic of fracture healing, to a regenerative process. [5] Evaluation of mechanotransduction mechanisms has demonstrated that tensile forces increase the expression of bone morphogenic proteins by osteoblasts and stimulate intramembranous bone formation. [7] This regenerative effect of gradual traction on tissue growth was originally designated by Ilizarov as the Law of Tension-Stress. [8] The process of distraction osteogenesis incorporates 3 major phases. The Latency phase is the period Which starts immediately subsequent to the creation of osteotomy and lasts till the commencement of distraction. This delay allows for tissue organization and formation of callus which bridges the gap between the two osteotomized bone surfaces. [5], [9], [10] Aronson et al conducted an animal study to test the outcome of different latency durations on bone regenerations. [11] Contrasting to other concurrent studies, it was observed that bone formation was most reliable when no latency period was considered prior to distraction; hence the suggestion that latency phase may not be essential. A recent study appraising the benefits of the latency period on the outcomes of dentoalveolar DO proved that although a latency period did not enhance the amount and density of the newly formed bone, it slightly increased bone maturation. However, it was assumed that despite the minor effect of the latency period on the regenerated bone, this phase may be crucial for soft tissue regeneration. [12] Regardless of the existing controversy on the importance of the latency phase, a review on the corresponding literature demonstrated that a 5-7 day latency period is the most recommendable protocol for the various indications of craniofacial DO. [1] The second phase known as the Distraction period is characterized by the application of distraction forces. Histologically, this phase begins with configuration of a Fibrous Inter-Zone (FIZ) at which dense fibers of collagen demonstrate a longitudinal arrangement parallel to the direction of the distraction forces. In between the collagen bundles osteoblastic activity creates a zone of Micro-Column Formation (MCF). The two ends of the FIZ are characterized by areas of primary mineralization, thus dubbed as Primary Mineralization Front (PMF). [5], [10], [13] The distraction phase continues with active synthesis of fibrous tissue in central areas and active mineralization at the ends, till the acquired amount of elongation is gained. [14] As the major features of the distraction phase, Ilizarov underscored the significance of the rate and rhythm of distraction forces on the quality and quantity of the newly regenerated bone. The rate and the rhythm, respectively defined as the speed and the frequency of the applied distraction forces were assessed in an animal study. The results, reported in 1989, suggested distraction of $1 \mathrm{~mm}$ per day applied as $0.25 \mathrm{~mm}$ per 6 hours as the ideal distraction rate and rhythm for limb elongation. This was while slower distraction rates led to premature consolidation of the bone and faster distraction was accompanied by hindrance of osteogenesis. Moreover, he claimed that more satisfying results were obtained when distraction forces were applied with higher frequencies. [15] The current standard protocols of craniofacial DO seem to apply distraction forces at a rate of $1 \mathrm{~mm}$ per day 2-3 times. Nevertheless, these features may be altered in different patients. [1] Subsequent 
to the cessation of distraction, the third phase, designated as the Consolidation phase begins. This stage of distraction osteogenesis is distinguished by the growth of mineralization in a centripetal pattern. Moreover, transverse bridging connects the micro-columns of bone in the IFZ, leaving a honeycomb appearance. By the end of this phase, bone is being remodeled into a more mature lamellar bone, strong enough to function. [10] The duration of this period can be determined based on the amount of distraction. One month consolidation has been suggested for each centimeter of distraction. [16]

\section{Mandibular distraction osteogenesis}

The primary attempts for mandibular distraction osteogenesis date back to the 1970s when animal studies were designed to restore surgically shortened canine mandibles via gradual distraction. [17], [18] McCarthy et al were the first to apply gradual distraction for lengthening the human mandible. [4] The preliminary report of their study presented four children who underwent unilateral and bilateral mandibular expansion for management of unilateral microsomia and Nager's syndrome, respectively; 18 to $24 \mathrm{~mm}$ of mandibular distraction was achieved. Common indications for mandibular distraction are summarized in Table 1.

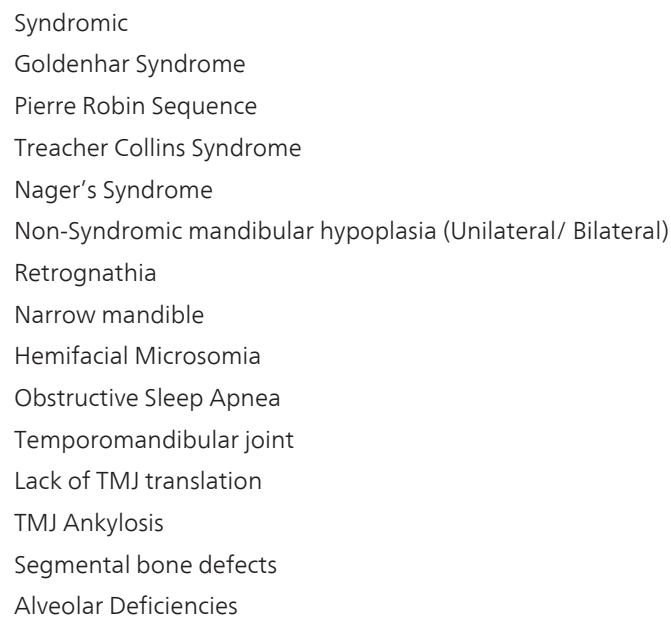

Table 1. Common indications for mandibular distraction osteogenesis

\subsection{Mandibular lengthening}

Mandibular hypoplasia, a condition associated with length deficiency in the mandibular ramus or/ and body, is a manifestation of impairment of mandibular growth caused by syndromic or non-syndromic congenital conditions or as a result of trauma. Depending on the severity of the deficiency, mild to severe esthetic and functional problems arise which obligate 
intervention. Mandibular hypoplasia has been traditionally managed by bone grafting, orthognathic surgery, and orthodontic therapy. These treatment approaches may contribute to considerable morbidity and unsatisfactory results in many situations, not to mention instances in which treatment appears unfeasible. The advent of craniofacial distraction osteogenesis has provided an alternative treatment modality to eliminate the shortcomings of conventional protocols for management of this craniofacial discrepancy. [4] In correction of mandibular hypoplasia in angle's class II patients where great amounts of advancement $(>10$ $\mathrm{mm}$ ) are required, DO has shown promising results with negligible relapse. This is probably due to the simultaneous expansion of the surrounding soft tissue. [19] In comparison, the bilateral sagittal split osteotomy, the most common treatment choice, not only provides less stable results with advancements larger than $6 \mathrm{~mm}$, but is also likely to be accompanied by serious adverse events, namely neurosensory disturbance of inferior alveolar nerve and disorders of the temporomandibular joint. [20], [21] On the other hand, bone grafting is another common choice of treatment for severe hypoplastic mandibles. [22], [23] Yet, the procedure seems not to be very desirable, since it may be associated with donor site morbidity and resorption of the graft. Another important indication for mandibular distraction osteogenesis is a lack of condylar translation. In growing patients with mandibular hypoplasia in whom condylar translations occurs normally during mandibular movements, functional orthodontic treatment may be of greater merit for restoration of the deficiency. Nevertheless, DO is a technique-sensitive procedure and demands patient compliance. Therefore, until randomized controlled trials have proved it beneficial over other treatment options for mandibular hypoplasia, it remains an alternative rather than a replacement for the existing treatment modalities. [20], [21]A variety of distraction devices have been designed and introduced for clinical implications; each associated with pros and cons. Extraoral distractors which are fixed in place by transcutaneous pins, are generally easier to manipulate and allow for multidirectional distraction. However, the psychosocial problems consequent to the presence of the device as well as facial scarring led to the emergence of intraoral distractors. [24], [25] McCarthy introduced the first intraoral distraction device in 1995. [26] Intraoral distractors are of three types: tooth-borne, bone-borne and hybrid distractors. [25] Finite element analysis of intraoral distraction devices demonstrated the hybrid type to be the most stable under masticatory loads, while tooth-borne distractors were the most reliable in transferring the expansion to the bone. [27] The morbidity associated with bone-borne devices appear to be higher than toothborne distractors. [28] While tooth-borne devices seem beneficial as they facilitate subsequent tooth movement, concerns such as greater mandibular expansion at the alveolar section comparing to the basal bone may arise with this type of distractor. [25] Shetye et al demonstrated that application of intraoral distractors may be associated with higher incidence of minor adverse events, with no effect on treatment outcome. This is while the occurrence of major incidents is more likely when extraoral distraction devices are used. [29]Mandibular hypoplasia is generally divided into two groups of unilateral and bilateral hypoplasia. A metaanalysis of mandibular distraction osteogenesis demonstrated the most common indication for unilateral DO to be hemifacial/craniofacial microsomia. [30] Unilateral craniofacial microsomia is a genetic disorder that affects the derivatives of the first and the second brachial arch and is initially characterized by abnormal growth of the mandibular ramus. The asym- 
metric growth of the mandible may gradually affect the growth of the surrounding structures, a fact that encourages surgeons to begin treating patients at early ages. The resultant facial asymmetry has been corrected via unilateral DO particularly in growing children. [31], [32] The authors analyzed the posteroanterior cephalometric changes subsequent to unilateral distraction osteogenesis in 10 patients. [33] Improvements in the piriform angle, intergonial angle, and the occlusal cant revealed the influence of the treatment on the maxillary and midfacial growth. It is highly suggested that the treatment be continued with functional orthodontic therapy in growing children. Functional appliances can act to obtain symmetry during growth. We have performed the combination of distraction osteogenesis and functional orthodontic therapy in a group of our patients. [34] It is advisable that the patient be followed until the end of growth and if necessary the functional orthodontic therapy be continued.

\subsubsection{Lengthening for asymmetry}

\section{Case 1}

An 8-year-old male patient with a history of right condylar trauma at birth presented with facial asymmetry, cant of the occlusal plane, deviation of the midline, and a deep-bite malocclusion (Figure 1-A, B, C). A horizontal osteotomy was made at the body of the right ramus below the mandibular foramen and a custom-made unidirectional extraoral distractor was fixed in place (Figure 1-D, E). Following a 7-day latency period, the distractor was activated at a rate of $1 \mathrm{~mm} /$ day. Distraction was stopped after the ramus was elongated by $22 \mathrm{~mm}$ (Figure 1-F). Subsequent to removal of the distractor a hybrid functional appliance was used to manage the posterior right open-bite created as a result of mandibular lengthening (Figure 1-G, H). Functional therapy continued for 3 years when fixed orthodontic therapy was initiated in order to restore the position of impacted left canine (Figure 1-I, J).

\subsubsection{Unilateral mandibular hypoplasia}

\section{Case 2}

A 6-year-old male patient with a history of trauma at age 2, presented with facial asymmetry and midline deviation due to unilateral mandibular hypoplasia (Figure 2-A, B). A horizontal osteotomy was done in the right ramus and a unidirectional intraoral distractor (KLS Martin, Tuttlingen, Germany) was fixed in place (Figure 2-C). Distraction was initiated with an oblique vector (Figure 2-D). Consequently, along with a posterior open bite, the teeth were deviated to the opposite side to a considerable extent (Figure 2-E). This was corrected via cross elastic traction (Figure 2-F). The patient was followed during growth and no deviation or facial asymmetry occurred; hence no need for further orthodontic treatment.

\subsubsection{Hemifacial microsomia}

\section{Case 3}

A 9-year-old male patient with hemifacial microsomia type 2 A was planned to receive distraction osteogenesis for treatment of facial asymmetry (Figure 3-A, B). Mandibular ramus 


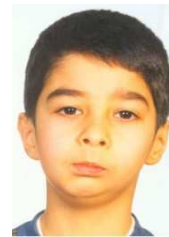

(a)

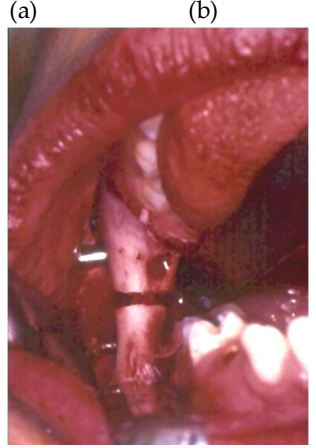

(d)

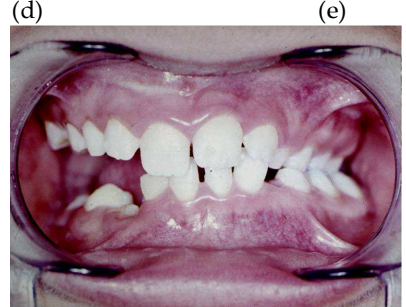

(g)

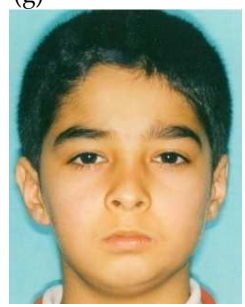

(i)

(j)
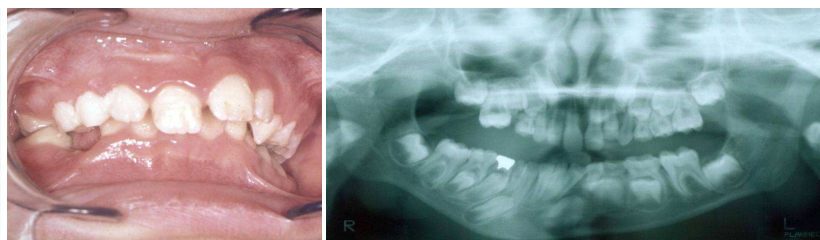

(c)
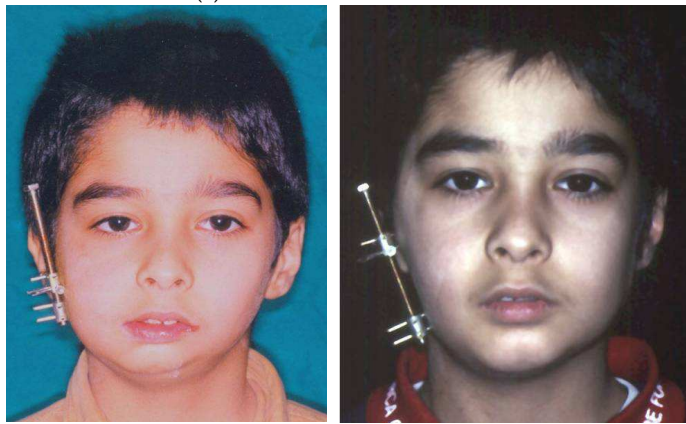

(f)

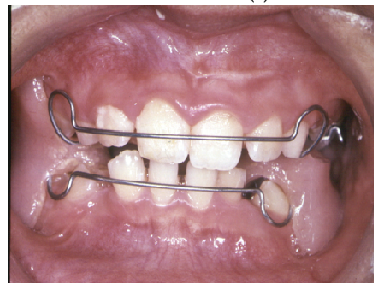

(h)

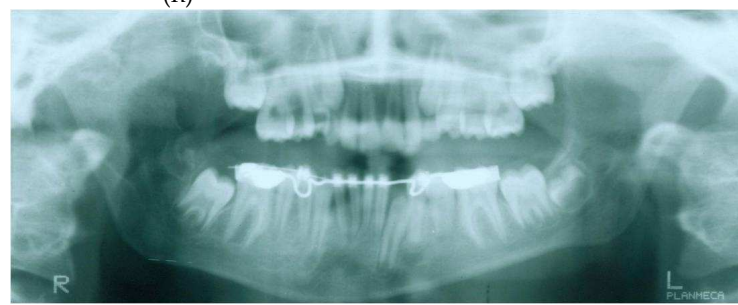

Figure 1. (a) Pre-distraction facial appearance. Facial asymmetry and cant of occlusal plane is apparent. (b) Pre-distraction intraoral view. (c) Panoramic view. (d) Horizontal osteotomy was made at the body of the right ramus below the mandibular foramen. (e) A custom-made unidirectional extraoral distractor was fixed in place. (f) Ramus was elongated by $22 \mathrm{~mm}$. (g) The posterior open-bite was created at the right side as a result of mandibular lengthening. ( $h$ ) A hybrid functional appliance was used to manage the posterior right open-bite. (i) Facial appearance 3 years post-distraction. (j) Panoramic view 3 years post-distraction.

was elongated using an extraoral distractor. Following a 2-month consolidation period, the distractor was removed. Orthodontic functional therapy was started to correct the posterior open bite (Figure 3-C, D). Orthodontic therapy was continued for 5 years (Figure 3-E, F). 


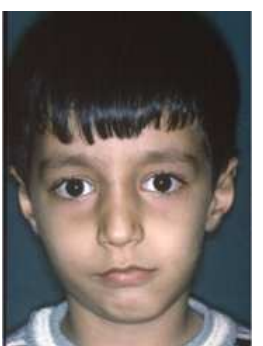

(a)

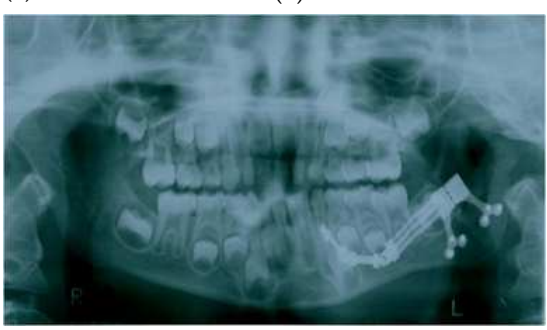

(d)

(b)
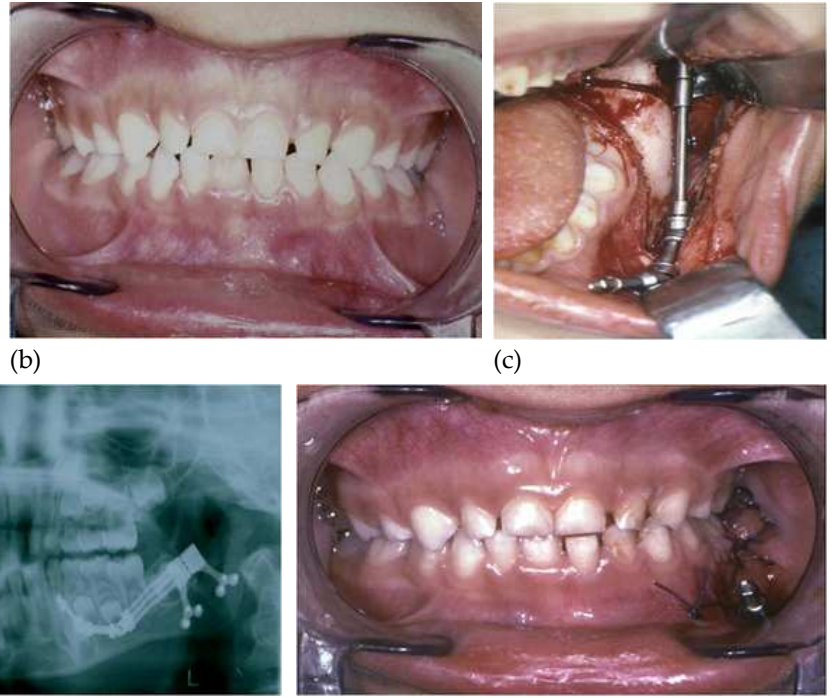

(e)

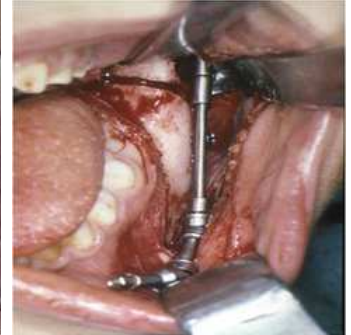

(c)

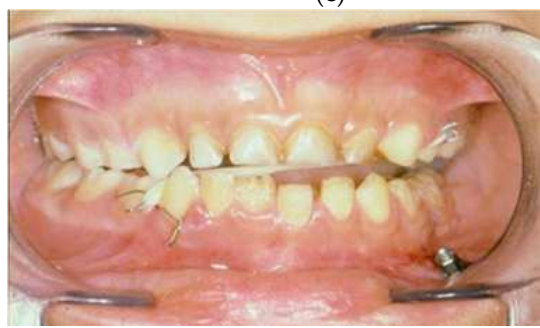

(f)

Figure 2. (a) Pre-distraction facial appearance demonstrates facial asymmetry due to childhood trauma. (b) Intraoral view shows midline deviation. (c) A horizontal osteotomy was made at the body of the right ramus and an intraoral distractor was fixed in place. (d) Distraction was initiated with an oblique vector. (e) Post-distraction intraoral view. Teeth were deviated to the opposite side. (f) Deviation was corrected via cross elastic traction.

\subsubsection{Hemifacial microsomia}

\section{Case 4}

A 17 year-old female patient with hemifacial microsomia presented with facial asymmetry and midline deviation to the right. The right maxillary canine was impacted (Figure 4-A-E). Predistraction orthodontic therapy included maxillary expansion and repositioning the impacted canine into the arch (Figure 4-F, G). Subsequently, unilateral osteotomy in the ramus was performed and an extraoral distraction device was fixed in place. With a rate of $1 \mathrm{~mm}$ per day, distraction was continued until adequate elongation was obtained (Figure 4-H-K ). Fixed orthodontic treatment was ongoing during the consolidation period (Figure 4-L). Final maxillary and mandibular arch coordination was achieved through bimaxillary orthognathic surgery (Figure 4-M-S). 


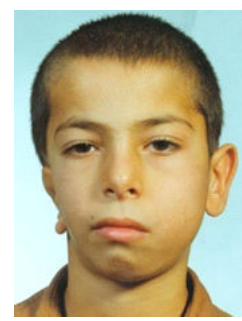

(a)

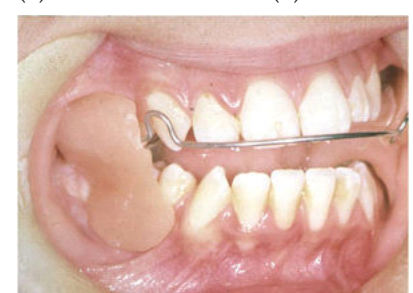

(d)

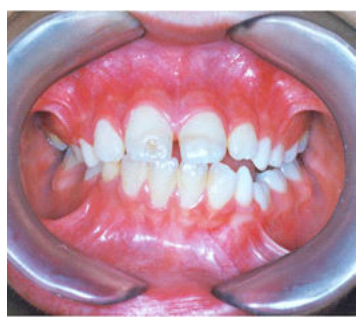

(b)

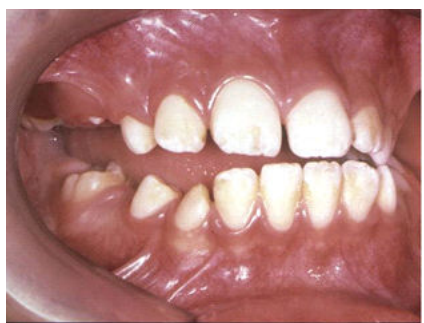

(c)

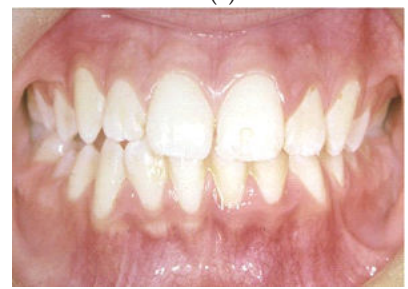

(e)

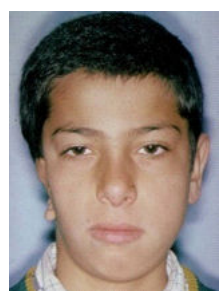

(f)

Figure 3. (a) Pre-distraction facial asymmetry due to hemifacial microsomia. (b) Pre-distraction intraoral view. (c) Postdistraction intraoral view. Unilateral posterior open was created. (d) Orthodontic functional therapy was started to correct the posterior open bite. (e) Five years post-distraction intraoral view. (f) Facial appearance 5 years post-distraction.

\subsubsection{Facial asymmetry}

\section{Case 5}

A 13-year-old female patient presented with mandibular deformity due to left condylar ankylosis (Figure 5-A). The patient had received a costochondral graft at age 6 and the function of the joint was restored (Figure 5-B). The remaining facial asymmetry was planned to be resolved via distraction osteogenesis. Using an extraoral custom-made distraction device, the left ramus was elongated by $18 \mathrm{~mm}$ (Figure $5-\mathrm{C}$ ). The resultant posterior open bite was corrected via 3 years of hybrid functional therapy followed by fixed orthodontic treatment (Figure 5-D, E, F).

\subsubsection{Mandibular asymmetry due to condylar ankylosis}

\section{Case 6}

A 16-year-old female patient presented with mandibular asymmetry due to left condylar ankylosis (Figure 6-A, B). At age 8, the patient had undergone a condylectomy procedure for treatment of the condylar ankylosis. She was then a candidate for distraction osteogenesis. Elongation of the left ramus $(20 \mathrm{~mm})$ was achieved by an extraoral distraction device (Leibinger Multiguide, Freiburg, Germany) (Figure 6-C, D). Eight months following removal of the distractor, the patient was orthodontically prepared for an orthognathic surgery. The surgery included Le Fort I and bilateral sagittal split osteotomies as well as genioplasty. A normal class I occlusion was obtained (Figure 6-E, F). 

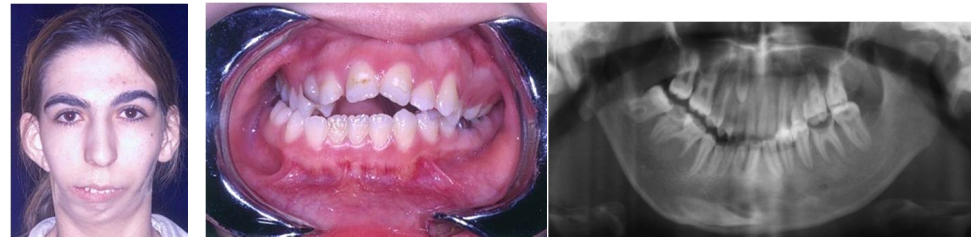

(b)

(c)

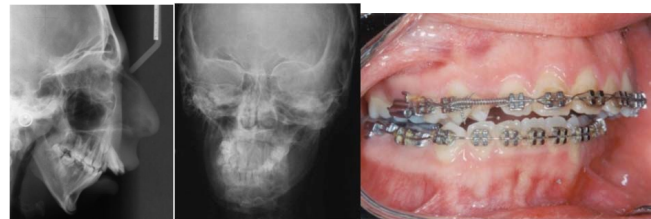

(d)

(e)

(f)

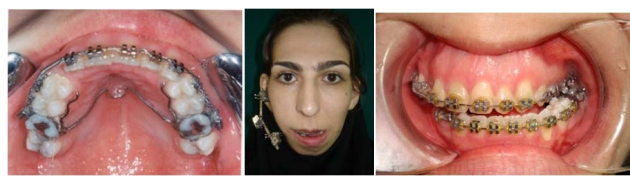

(g)

(h)

(i)

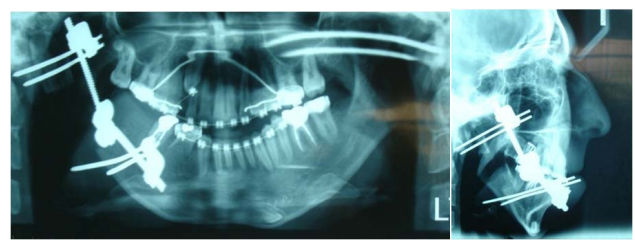

(j)

(k)

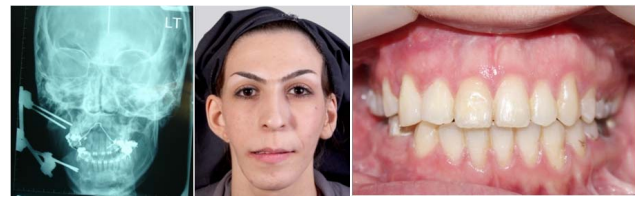

(1)

(m)

(n)

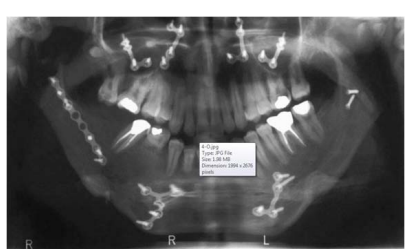

(o)

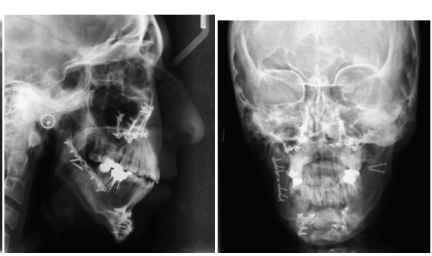

(q)

Figure 4. (a) Pre-distraction facial asymmetry. (b) Pre-distraction intraoral view. (c) Pre-distraction panoramic view. (d) Pre-distraction lateral cephalometric view. (e) Pre-distraction posteroanterior (PA) cephalometric view. (f) Orthodon- 
tics included maxillary expansion. (g) The impacted canine was brought into the arch. (h) Post-distraction facial appearance. (i) Post-distraction intraoral view. (j) Post-distraction panoramic view. (k) Post-distraction lateral cephalometric view. (I) Post-distraction PA cephalometric view. (m) Post-orthognathic surgery facial appearance. (n) Post-orthognathic surgery intraoral view. (o) Post-orthognathic surgery panoramic radiograph. (p) Lateral cephalometric radiograph. (q) Post-orthognathic surgery PA cephalometric radiograph.

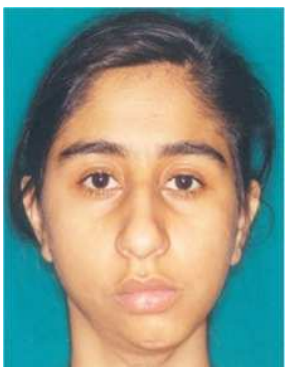

(a)

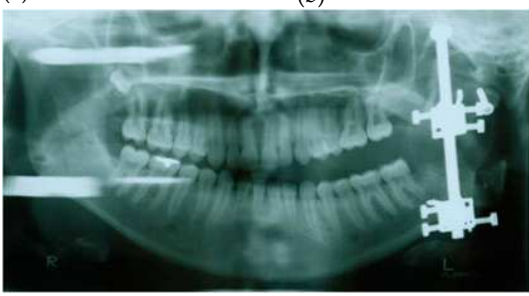

(c)

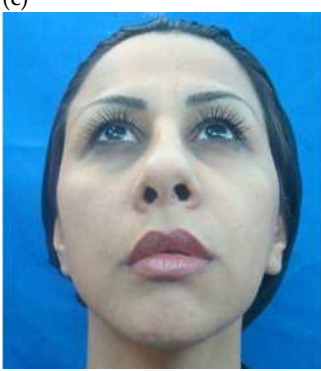

(e)

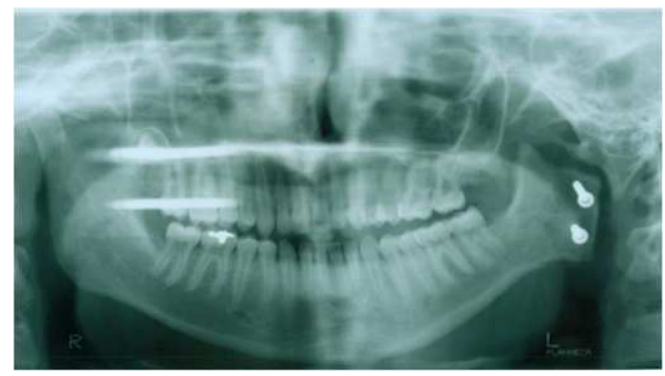

(b)

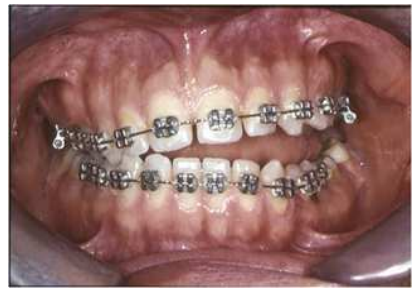

(d)

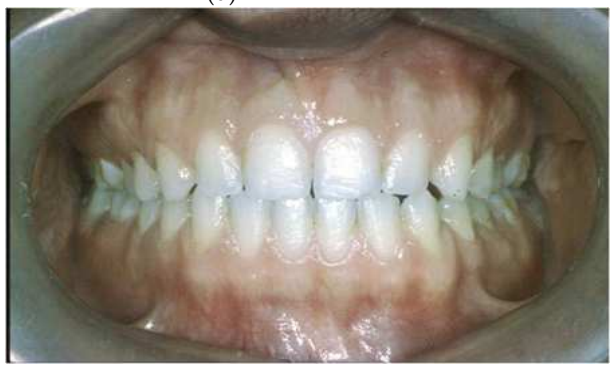

(f)

Figure 5. (a) Pre-distraction facial asymmetry. (b) Pre-distraction panoramic view. Bone screws remained from a previous costochondral bone graft can be observed. (c) Immediate post-distraction panoramic view. Mandibular ramus elongated by $18 \mathrm{~mm}$. (d) Posterior open bite was corrected via functional therapy and fixed orthodontic therapy. (e) Six years post-distraction facial appearance. (f) Normal occlusion was obtained.

\subsection{Bilateral hypoplasia}

Similar to unilateral mandibular hypoplasia, several etiologies are documented for bilateral hypoplasia including syndromic conditions, condylar fracture due to trauma, and class II malocclusion. Along with undesirable facial appearance and disorders in the masticatory system, micrognathia which itself may be symmetric or asymmetric, can cause mild to lethal 

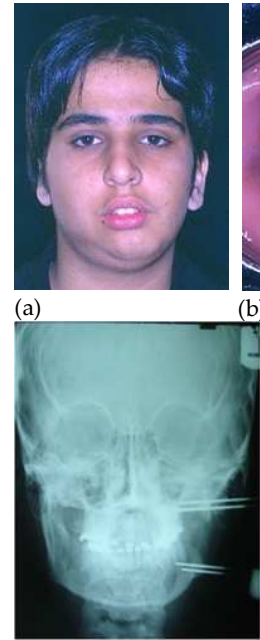

(d)

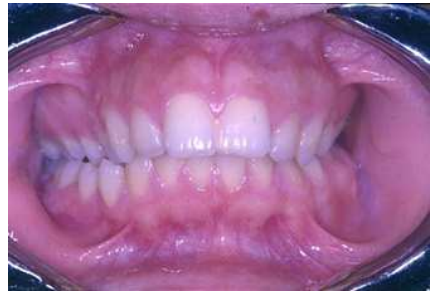

(b)

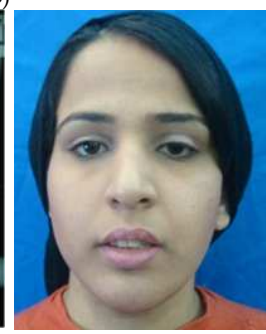

(e)

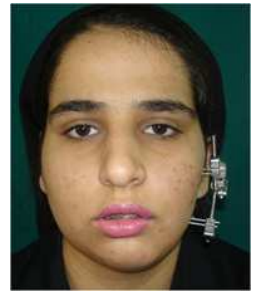

(c)

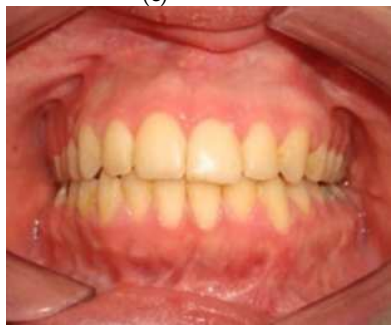

(f)

Figure 6. (a) Pre-distraction facial appearance. (b) Pre-distraction intraoral view. (c) Extraoral distractor was used for mandibular lengthening. (d) Left ramus was elongated by $20 \mathrm{~mm}$. (e) Two years post-distraction facial appearance. Orthognathic surgery has been performed. (f) Normal occlusion has been obtained.

degrees of airway obstruction. [35] Havlik and Bartlett [36] as well as Moore and co-workers [37] were the first to apply distraction osteogenesis for management of micrognathia. A metaanalysis indicated Pierre Robin sequence as the most common condition treated with bilateral DO. [30] Pierre Robin syndrome is a congenital anomaly characterized as a triad of micrognathia, glossoptosis, and cleft palate. [38] Obstructive sleep apnea; recognized in severe degrees of the syndrome, implicates intervention at early ages. Severe airway obstruction which may also be a manifestation of temporomandibular joint ankylosis [39] is traditionally treated with tracheotomy. This invasive intervention although remains to be the gold standard, has been associated with considerable morbidity. [40] Mandibular DO allows for early treatment in neonates and infants. It is noteworthy that despite the promising results accomplished with DO at early ages [40]- [42], long-term follow-ups are required to evaluate the stability of the outcomes.

\subsubsection{Severe mandibular deficiency}

\section{Case 7}

A 6-year-old boy presented with severe mandibular deficiency. The patient suffered from obstructive sleep apnea (Figure 7-A-F). Prior to distraction osteogenesis, orthodontic treatment was done and included maxillary arch expansion with a quad-helix appliance followed by application of an anterior bite plate (Figure G). Subsequently, bilateral distraction osteogenesis was performed via extraoral multi-guide distraction devices (Leibinger, Freiburg, Germany). The amount of elongation obtained at the end of the distraction phase was about $32 \mathrm{~mm}$; 
though not equal on both sides (Figure H-J). Obstructive sleep apnea was completely resolved in this patient. Treatment was continued with functional orthodontic therapy; however, the patient was only followed for 2 years (Figure K-P).

\subsubsection{Mandibular deficiency}

\section{Case 8}

A 14-year-old patient presented with skeletal class II malocclusion and severe deep bite (Figure 8-A, B). The deficiency was planned to be corrected by distraction osteogenesis. Bilateral horizontal osteotomies were made in the body of the ramus. Unidirectional intraoral distractors (KLS Martin, Tuttlingen, Germany) were fixed in place (Figure 8-C). Following mandibular lengthening for $20 \mathrm{~mm}$, an anterior open bite was created which could be attributable to improper distraction vector, a common adverse event with unidirectional distraction devices (Figure 8-D). This problem was solved by elastic traction (Figure 8-E) and normal occlusion was obtained. The patient has now been followed for 8 years (Figure 8-F, G).

\subsection{Mandibular widening}

Transverse mandibular deficiency is a common clinical problem, diagnosed by a narrow, Vshaped arch and anterior dental crowding. This problem may occur as an isolated condition, a component of certain syndromes [43], or a consequence of symphyseal fracture and tissue loss. [25], [44] Depending on the amount of the deficiency, various treatment protocols are available for mandibular arch expansion. The use of Arch wires, Schwarz plates, lingual arches and functional appliances has been hampered to some extent by the limited stability of the accomplished results. On the other hand, tooth extraction or interdental stripping, more commonly indicated for adult patients, may not provide adequate space in severe cases. [44], [45] Management of extreme transverse deficiencies was conventionally achieved via osteotomy and placement of bone grafts. Attempting to rectify the possible adverse events of bone grafting, Guerrero first used symphyseal distraction osteogenesis for mandibular widening and called it "rapid surgical mandibular expansion". [46] This technique holds promising potential for expansion of the mandibular basal bone. More predictable results can be obtained in a shorter treatment period. Yet, the probable relapse of the treatment gains is a major concern for surgeons. The possibility of teeth proclination, nonhomogeneous dental and skeletal expansion, as well as device-related difficulties should also be taken into consideration. [47] Based on the literature, symphyseal distraction osteogenesis has been suggested for patients above 12 years old. [1] Chung and Tae evaluated dental stability in an average 1.5 year followup duration subsequent to symphyseal distraction osteogenesis. By following the changes of 13 landmarks on study models, it was demonstrated that the total amount of surgical expansion did not decrease by relapse. [47] Both extraoral and intraoral distraction devices can be used for symphyseal distraction osteogenesis. Intraoral devices are more esthetically appealing. Though, as suggested by Kita et al, when extremely narrow mandibles are to be expanded, placement of intraoral devices may not be feasible due to inadequate space. Moreover, the design of intraoral distractors does not allow for large amounts of expansion. Kita et al used symphyseal distraction osteogenesis via extraoral devices to treat extreme transverse man- 


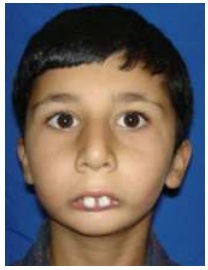

(a)

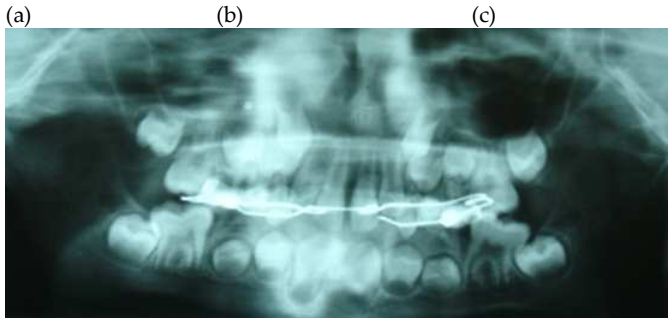

(d)

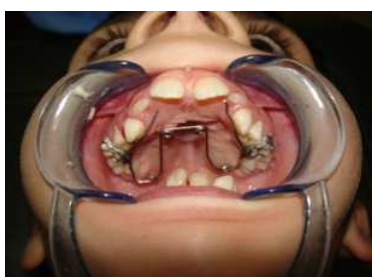

(g)

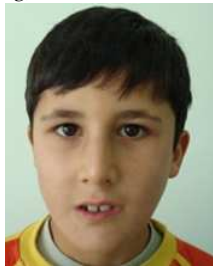

(k)

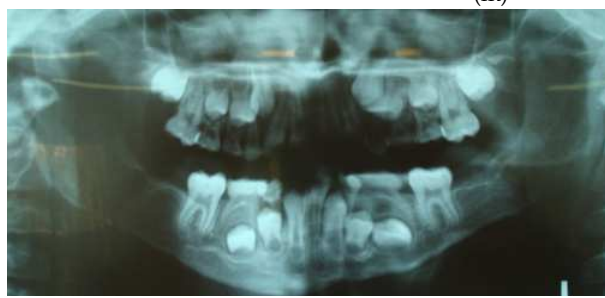

(n)
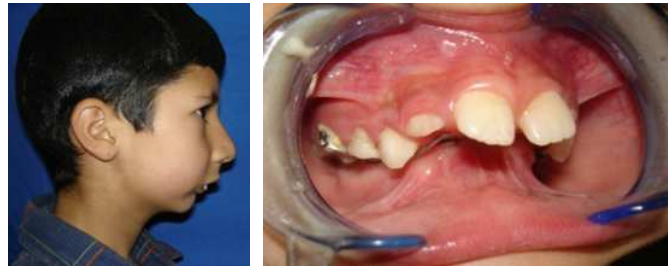

(c)

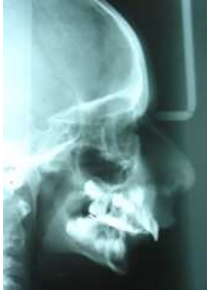

(e)

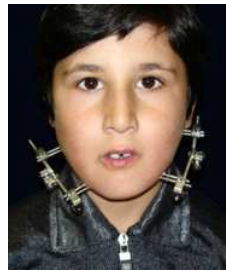

(h)

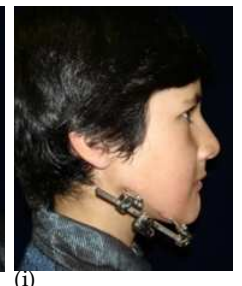

(f)
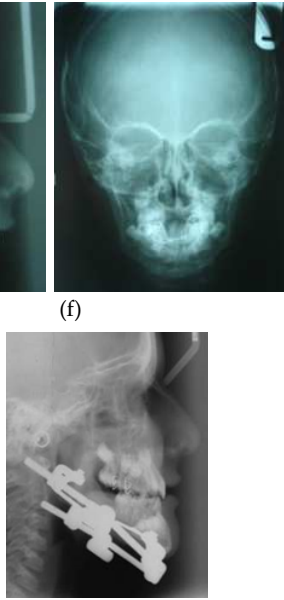

(j)

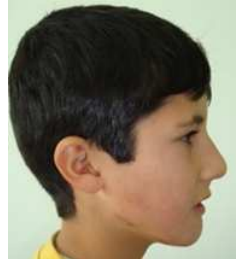

(1)

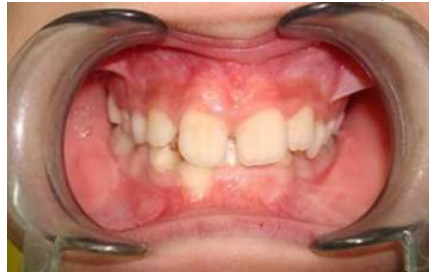

(m)

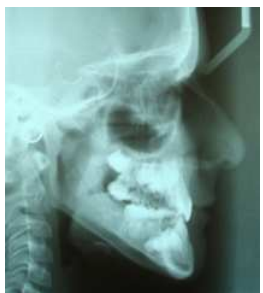

(o)

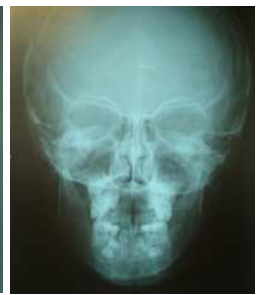

(p)

Figure 7. (a) Pre-distraction facial appearance. (b) Profile view. (c) Intraoral view. (d) Pre-distraction panoramic view. (e) Pre-distraction lateral cephalometric view. (f) Pre-distraction posteroanterior cephalometric view. (g) Pre-distraction orthodontic treatment included maxillary expansion via a quad-helix appliance. (h) Post-distraction facial appearance. (i) Profile view. (j) Lateral cephalometric view. (k) Two years post-distraction appearance. (I) Profile. (m) Intraoral view. (n) Two years post-distraction panoramic view. (o) Post-distraction lateral cephalogram. (p) Two years post-distraction PA cephalogram. 


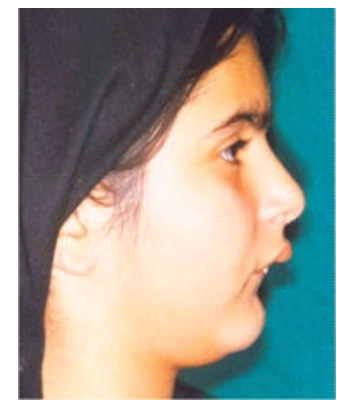

(a)

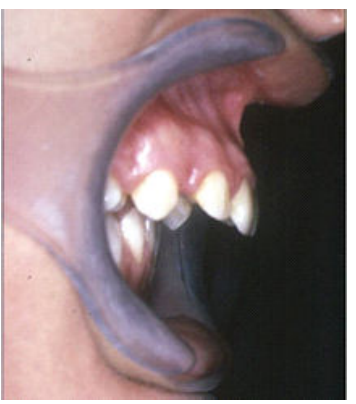

(b)

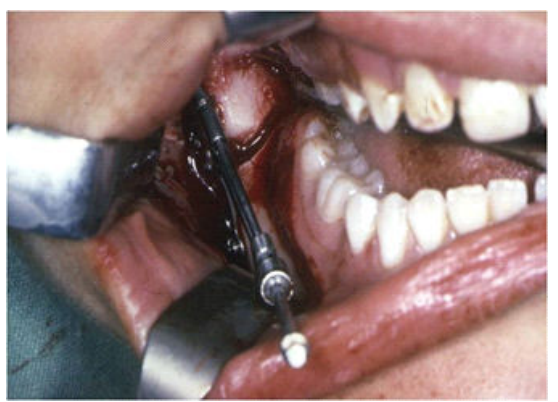

(c)

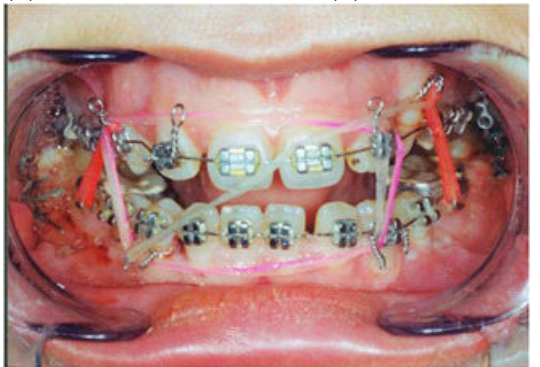

(d)

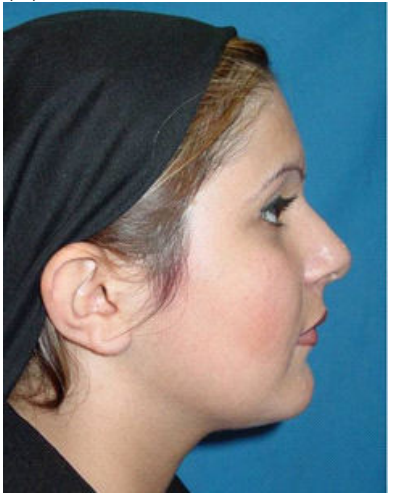

(f)

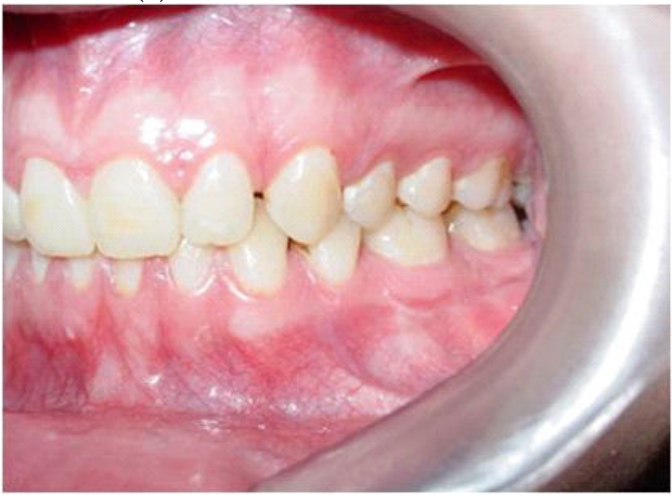

(g)

Figure 8. (a) Pre-distraction facial appearance. (b) Pre-distraction intraoral view. (c) Unidirectional intraoral distractors were fixed in place. (d) Following mandibular lengthening for $20 \mathrm{~mm}$, an anterior open bite was created. (e) Anterior open bite was corrected by elastic traction. (f) Eight years post-distraction facial appearance. (g) Intraoral view.

dibular deficiencies in patients with hypoglossia-hypodactyly syndrome. [43]A plethora of investigations and modifications have attributed to enhanced efficiency of mandibular distraction osteogenesis. Yet, the technique is not exempt of adverse events. Diverse rates of incidence have been reported for different mandibular distraction osteogenesis procedures. Shetye et al [29] classified the potential adverse events associated with mandibular DO into three groups: minor incidents indicated those with no influence on the outcome. Moderate and 
major incidents were both defined as events that result in undesirable outcome and can or cannot be resolved via invasive procedures, respectively. Their 16 year follow-up of 141 patients who underwent mandibular DO for unilateral or bilateral mandibular lengthening demonstrated that minor and moderate incidents were reported in $26.99 \%$ and $20.35 \%$, respectively; while in only $5.31 \%$ of patients did major events occur. The majority of major incidents included TMJ ankylosis and derangements as well as fibrous union. Nevertheless, taken all the above-mentioned complications into considerations, investigators seem to be unanimous in the safety of distraction osteogenesis.

\subsection{Maxillary distraction osteogenesis}

The Principles of distraction osteogenesis have been applied for correction of transverse and sagittal discrepancies of the maxilla and the midface associated with orofacial clefts and several syndromes. Midfacial distraction osteogenesis was first evaluated in animal studies performed on sheep [48] and dogs [49]. A preliminary human report of maxillary and midfacial advancement through the application of a distraction device was published by Cohen et al in 1997. [50] Two children with cleft lip and palate, midfacial hypoplasia, and class III malocclusion underwent treatment with distraction osteogenesis. Up to $11 \mathrm{~mm}$ advancement of the midfacial complex was achieved in both patients. Two years later, Mommaerts introduced a technique for maxillary expansion using a transpalatal distractor. [51] In comparison to rapid palatal expansion, the treatment protocol most frequently used for maxillary expansion, palatal distraction osteogenesis was asserted to eliminate particular adverse events such as alveolar bending, tooth tipping, buccal cortex fenestration, and relapse. Common indications for maxillary and midface distraction osteogenesis are summarized in Table 2.

Orofacial Clefts
Craniosynostosis
Crouzon's Syndrome
Apert's Syndrome
Pfeiffer Syndrome
Midface deficiencies of other causes
Alveolar deficiencies

Table 2. Common indications for maxillary and midface distraction osteogenesis.

\subsection{Maxillary and midfacial advancement}

The majority of cleft lip and palate patients suffer from degrees of maxillary hypoplasia, either as a primary manifestation of the cleft or secondary to attempts for cleft repair. This often complex discrepancy is conventionally corrected through a series of surgeries including different osteotomies. The inception of distraction osteogenesis for advancement of maxillarymidface in cleft patients brought new insight into the treatment of these patients. A metaanalysis of conventional osteotomies and distraction osteogenesis, along with many similarities between the two techniques, suggested distraction osteogenesis to be advanta- 
geous for it eliminates the need for bone grafts. [52] Moreover, it was demonstrated that most protocols postponed treatment with conventional osteotomies until growth was completed. In contrast, distraction osteogenesis was more frequently performed in growing patients; although, overcorrection was recommended to preclude relapse. Different types of extraoral and intraoral distractors have been established for maxillary distraction osteogenesis. Extraoral distractors have the capacity for multidirectional maxillary advancement and the vectors can be changed during the process. [53] Yet, many patients have difficulty accepting extraoral devices primarily due to the unappealing appearance and discomfort. [54] Moreover, the external position of these devices makes them prone to loosening and fracture following an accidental trauma. [53] Rigid external distraction (RED) device is fixed to the cranium. This allows for protection of maxillary teeth comparing to other types of extraoral devices which are anchored to the maxilla. [54] The stability of maxillary advancement with RED was evaluated in a 3-year prospective study. To avoid the possible interference of growth in the outcomes, the study was performed on adult patients. The relapse was reported to be $22 \%$ after 3 years. [55] Internal distractors cause less psychosocial problems for the patient and are less likely to be loosened or displaced during the distraction period or following traumatic forces. Moreover, being more easily tolerated by patients, an internal device can be maintained during the consolidation phase for as long as deemed necessary for prevention of relapse. [53], [54], [56] Nevertheless, installation of intraoral distractors may not be always feasible due to inadequate space. In addition, intraoral distractors provide unidirectional bone movement; hence demanding precise positioning. [53] Complications such as fracture and collapse of the cleft alveolar bone has been reported with intraoral devices used for Le Fort I distraction. [56] Picard et al described a rigid internal device (RID) with the ability to provide unrestricted lengthening for total or segmental advancement of the maxilla. In 19 syndromic, cleft, and traumatic patients treated with this distractor, an average advancement of $9.6 \mathrm{~mm}$ was achieved. [54] A retrospective study comparing extraoral and intraoral distractors for midface advancement in syndromic patients demonstrated no significant difference regarding the complication rate and amount of lengthening between the two types. Accordingly, both distractors were asserted to be safe and it was suggested that choosing a device be individualized based on each patients needs and toleration. [53]Mild to moderate cases of maxillary hypoplasia which were traditionally corrected via Le Fort I osteotomy, have been successfully treated with anterior maxillary distraction osteogenesis. [57] Le Fort I osteotomy shows a negative impact on velopharyngeal competence and speech while this problem is rarely seen with anterior maxillary distraction osteogenesis. [57] Nonetheless, more severe cases may necessitate Le Fort I distraction.

\subsubsection{Cleft lip and palate and class III malocclusion}

\section{Case 9}

A 17-year-old female patient with cleft lip and palate presented with a class III malocclusion and anterior and posterior cross bite (Figure 9 A-C). Following pre-distraction orthodontic treatment, maxillary advancement was performed through Le Fort I osteotomy and RED device (KLS Martin, Figure 9-D). Maxilla was advanced by $18 \mathrm{~mm}$ (Figure 9-E, F). Orthodontic 
treatment continued for a year. Meanwhile, the patient received a removable partial prosthesis to replace the anterior missing teeth. Subsequently, the patient underwent rhinoplasty and primary lip repair (Figure 9-G-J). The procedures for lip repair are still ongoing.
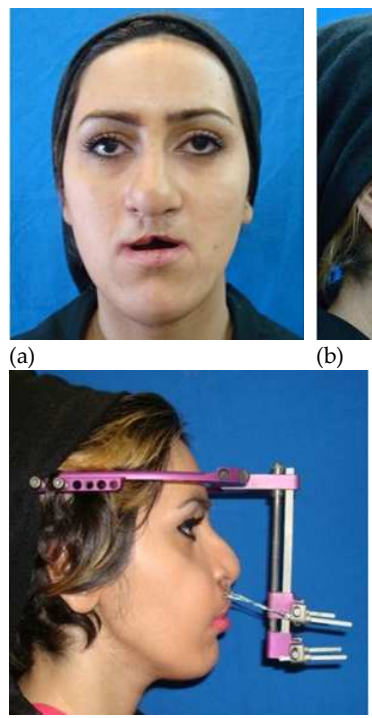

(d)

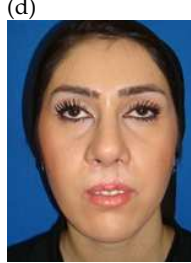

(g)
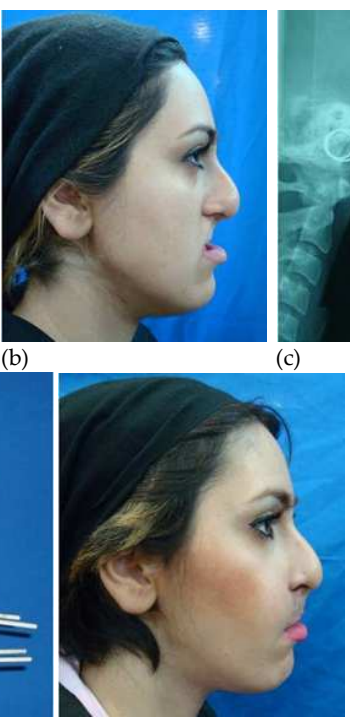

(e) (h)

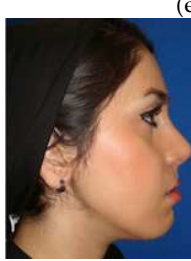

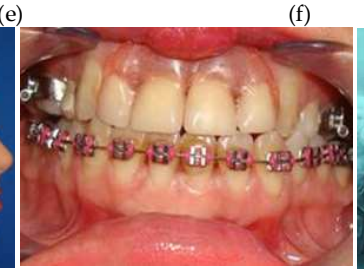

(i)

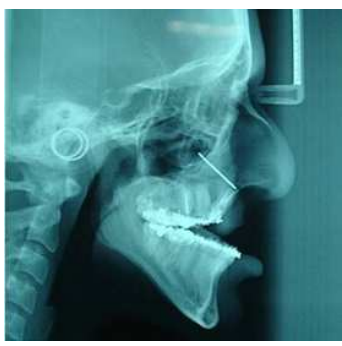

(c)
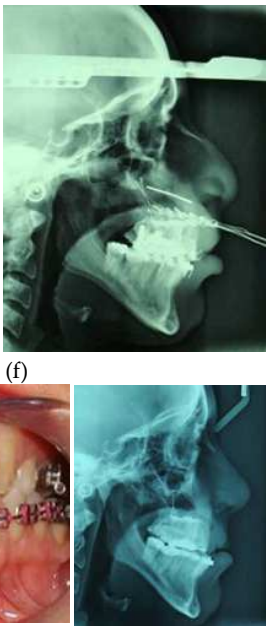

(j)

Figure 9. (a) Pre-distraction appearance. (b) Profile view. (c) Lateral cephalometric view. (d) Maxillary distraction osteogenesis was performed using an RED device. (e) Post-distraction facial appearance. (f) Post-distraction lateral cephalometric view. (g) Two years post-distraction facial appearance. (h) Profile. (i) Intraoral view. (j) Lateral cephalometric view.

Distraction osteogenesis has also proved valuable for treatment of patients affected with craniosynostosis. This condition caused as a result of premature fusion of cranial sutures is a clinical feature of particular syndromes such as Crouzon, Apert, and Pfeiffer. In the severe expression of these syndromes, it may be crucial to initiate treatment as early as 1 year of age. [58] Le Fort III and monobloc osteotomies are frequently used for management of craniosynostosis. [59] During the recent years, distraction osteogenesis has become popular for correction of syndromic maxillary hypoplasia. The amount of advancement of midface that can be achieved by distraction osteogenesis is generally greater than the amount obtained by conventional osteotomies such as Le Fort III and monobloc osteotomy. [60] Long-term follow 
ups of syndromic patients who underwent maxillary-midface advancement with distraction osteogenesis have proved the stability of the results. [59], [61], [62]

\subsection{Maxillary expansion}

Maxillary transverse deficiency is a condition associated with anterior and posterior dental crowding, unilateral or bilateral cross-bite, as well as TMJ and respiratory problems. Expansion of maxillary bone during growth is usually feasible through orthodontic treatments. However, with skeletal maturation, a combination of surgical and orthodontic techniques may be inevitable in order to accomplish adequate expansion. When the condition is accompanied with cleft lip and palate, it poses even greater challenges for treatment. Treatment of maxillary constriction can be performed by means of Le Fort I osteotomy and expansion. This protocol allows for multidirectional expansion of the maxillary complex; however, the resistance of the palatal fibromucosa diminishes the stability of the results. Another treatment option established for this deformity is surgically assisted rapid maxillary expansion (SARME). This technique eliminates soft tissue resistance via distraction histogenesis and can be based upon either tooth-borne or bone-borne devices. Tooth-borne distractors transfer forces to the teeth, leading to tooth-related adverse events such as root resorption, tooth tipping, and cortical fenestration. In contrast, bone-borne devices; first introduced by Mommaerts as a transpalatal distractor (TPD), are exempt of these undesirable effects for they directly apply forces to the bone. [63], [64] Application of bone-borne distractors becomes of paramount importance particularly when insufficient tooth support exists due to tooth missing and impaction. [65] Yet, they require a secondary surgery for removal. [63], [64] It is noteworthy that despite the disadvantages commonly considered for tooth-borne device, no considerable difference in dental tipping and stability has been found between tooth-borne and bone-borne maxillary distractors. [64], [66]

\subsubsection{Skeletal class III malocclusion and narrow maxilla}

\section{Case 10}

A 25-year-old female patient presented with dental and skeletal class III malocclusion and a narrow maxilla. Both maxillary and mandibular midlines had a shift to the right side. A 2-mm reverse over jet and anterior and posterior cross bites were present (Figure 10-A-D). Restriction in mandibular movement was found on examination. Treatment plan included SARPE via Smile distractor (Titamed). Transverse distraction was started at a rate of $1 \mathrm{~mm} /$ day and continued until $10 \mathrm{~mm}$ expansion was achieved (Figure 10-E, F). Post-distraction fixed orthodontic treatment closed the resultant gap between the two central incisors and repositioned the right lateral incisor into the dental arch (Figure 10-G-J).

\subsubsection{Maxillary transverse deficiency}

\section{Case 11}

A 20-year-old male patient presented with a class III malocclusion, maxillary transverse deficiency, severe anterior crowding, anterior open-bite, and bilateral cross-bite (Figure 11-A- 


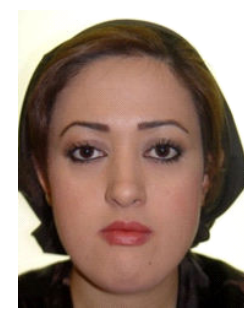

(a)

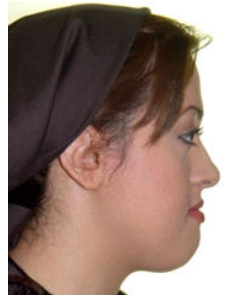

(b)

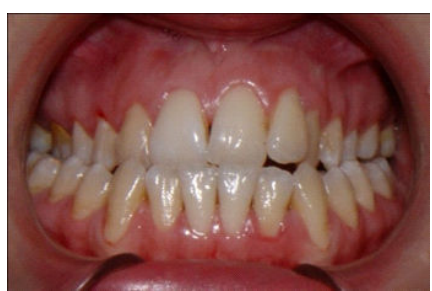

(c)

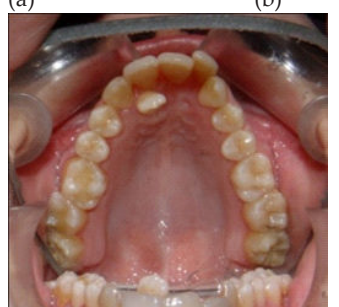

(d)

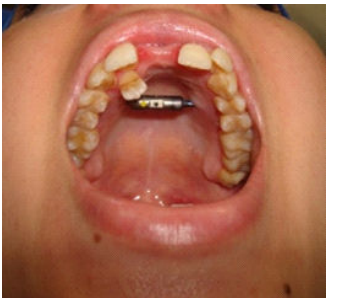

(e)

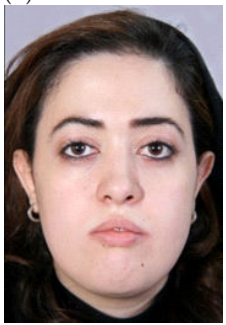

(g)

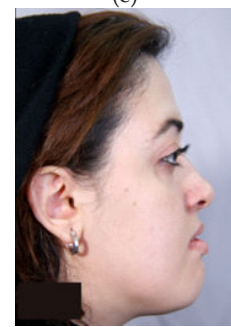

(h)

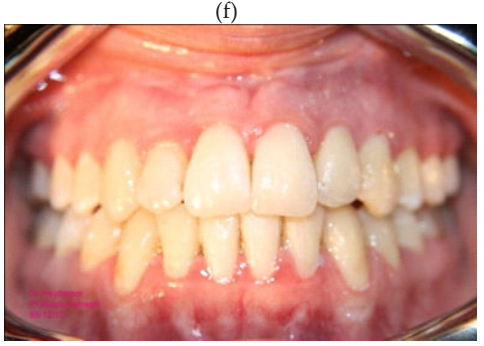

(i)

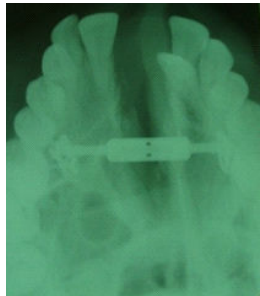

(f)

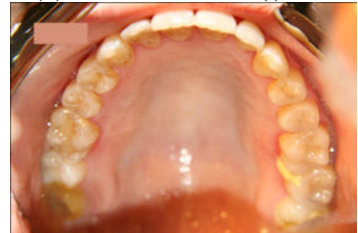

(j)

Figure 10. (a) Pre-distraction facial appearance. (b) Profile. (c) Intraoral view. (d) Pre-distraction intraoral view, the right lateral incisor is in a palatal position. (e) Post-distraction intraoral view. Maxilla expanded by 10mm. (f) Post-distraction radiograph. (g) Post-orthodontic treatment facial appearance. (h) Profile. (i) Intraoral view. (j) Post-orthodontic intraoral view. The right lateral incisor is repositioned into the arch.

E). The treatment plan included SARME with a bone-borne distractor followed by orthognathic surgery in order to respectively correct the transverse deficiency and the open bite. An osteotomy was made in the palatal midline, between the roots of the two central incisors and the distraction device (Smile distractor, Titamed) was placed (Figure 11-F-H). Following a 7day latency period, the distractor was activated at a rate of $1 \mathrm{~mm} /$ day. When expansion of 10 $\mathrm{mm}$ was achieved, activation was stopped and the device was maintained for a 2-month consolidation period (Figure 11-I). The device was kept for another 4 months until the space 
created between the central incisors was closed by orthodontic forces. Subsequently, the patient was orthodontically prepared for orthognathic surgery, Le Fort I osteotomy (Figure 11-K). Arch coordination was obtained. The patient is still under orthodontic treatment (Figure 11-L-P). It is worth mentioning that in this patient, alignment of maxillary teeth could have been achieved by extraction of premolars and fixed orthodontic therapy. However, this treatment protocol would impede maxillary and mandibular arch coordination. On the other hand, it is important that the amount of maxillary expansion is proportional to the mandibular arch.

\subsection{Alveolar distraction osteogenesis}

Alveolar distraction osteogenesis is pre-implant/ pre-prosthetic procedure which tends to restore the alveolar deficiencies and prepare the alveolar ridge for further rehabilitative treatments. Alveolar distraction osteogenesis was first evaluated in an animal model by Block et al. [67] Chin and Toth extended its application to human. [68] Alveolar ridge augmentation is frequently conducted via the use of different types of bone grafts. However, distraction osteogenesis not only decreases the complications and the duration of treatment, but also allows for reconstruction of large defects by simultaneously expanding the surrounding soft tissue. [69] Studies have suggested the amount of newly formed bone resorption prior to implant placement to be greater with onlay bone grafting in comparison to distraction osteogenesis. Peri-implant bone loss was comparable between the two techniques. [70], [71] On the other hand, the amount of augmentation gained with distraction osteogenesis was reported to be significantly greater than that obtained with inlay bone grafts. [72] Depending on the type and the extension of an alveolar defect, distraction osteogenesis may be considered either as an absolute treatment for reconstruction or as an adjunctive therapy along with other bone grafting procedures. Jensen and Block presented a classification of alveolar defects aiming to facilitated treatment planning with alveolar distraction osteogenesis. Accordingly, the more complex a defect, the greater the possibility of requiring bone grafts prior or subsequent to distraction osteogenesis. [73] This treatment modality can also be considered when previous attempts for bone grafting have failed. [74]

\subsubsection{Vertical alveolar distraction osteogenesis}

The technique of alveolar distraction osteogenesis have been successfully used for enhancing alveolar ridge height. [69], [74]- [81] The majority of studies evaluated the efficiency of distraction osteogenesis in the anterior parts of maxilla and mandible and the amount of obtained augmentation was reported between 5 to $15 \mathrm{~mm}$. Benefits of this method for augmentation of severely atrophic ridges remains to be a matter of controversy. Basal bone fracture and neurosensory complications have been suggested as the two most common problems associated with vertical distraction of atrophic mandibular ridges. [82] Indication of vertical alveolar distraction osteogenesis is therefore limited to areas where 5-7 $\mathrm{mm}$ of alveolar bone exists. [83] 

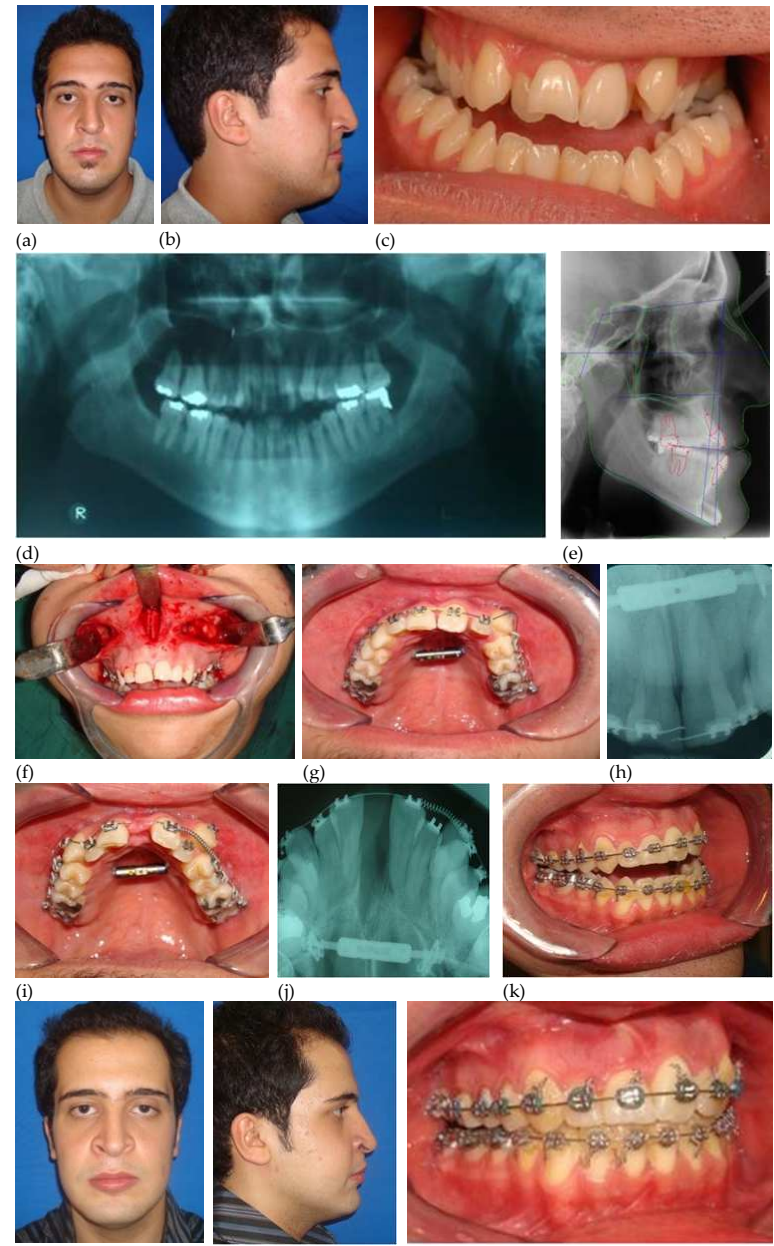

(1) $(\mathrm{m})$

(n)
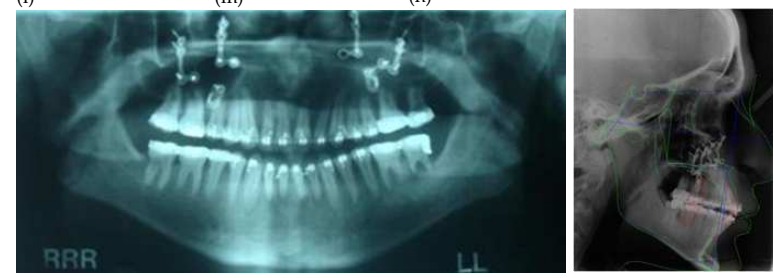

(o)

Figure 11. (a) Pre-distraction facial appearance. (b) Profile. (c) Intraoral view. (d) Pre-distraction panoramic view. (e) Pre-distraction lateral cephalometric view. (f) Osteotomy was made in the palatal midline. (g) Palatal distractor in place before activation. (h) Periapical radiography. (i) Two months post-distraction intraoral view. (j) Two months postdistraction occlusal radiograph. (k) Anterior open bite was planned to be corrected via orthognathic surgery. (I) Postorthognathic surgery facial appearance. $(m)$ Profile. $(n)$ Intraoral view. (o) Post-orthognathic surgery panoramic view. (p) Post-orthognathic cephalometric view. 
Distraction devices for alveolar distraction osteogenesis include both extraoral and intraoral devices as well as distraction implants. Extraoral distractors which are mainly positioned subperiosteally in the buccal vestibule, can only be used when a bone height of 6-7 $\mathrm{mm}$ is present. Comparing intraoral and extraoral distractors for alveolar distraction osteogenesis, Uckan et al demonstrated that the majority of complications associated with intraoral distractors were related to displacement and fracture of the transport segment. However, interference of the device with the opposing dental arch was considered the most frequent complication with extraoral distractors. [71] Distraction implants initially pose distraction forces to augment the alveolar ridge and are subsequently kept in place to act as a dental implant. These devices have the advantage of eliminating the need for a second surgery for distractor removal. Yet, it is highly likely that the ideal position of the distractor does not correspond to the desirable position of the implant. [81], [83]

\subsubsection{Alveolar deficiency}

\section{Case 11}

A 20 year-old female patient presented with a large bone defect in the anterior mandible due to resection of a central giant cell granuloma (Figure 12-A, B). The patient underwent alveolar distraction osteogenesis. Horizontal osteotomy was performed and an intraoral distractor (KLS Martin) was placed (Figure 12-C, D). Following a 7-day latency phase distraction was initiated at a rate of $1 \mathrm{~mm}$ per day. $18 \mathrm{~mm}$ augmentation was achieved (Figure 12-E). After the consolidation period, the distractor was removed and dental implants were inserted into the regenerated bone. Due to insufficient ridge width, a guided bone regeneration procedure was done to induce bone regeneration over the exposed surfaces of the implants (Figure 12-F). 3 months later, at the second stage of implant surgery inadequate keratinized tissue was compensated by a connective tissue graft from the palate (Figure 12-G-I). Fixed implantsupported prosthesis restored the missing teeth (Figure 12-J).

\subsection{Horizontal alveolar distraction osteogenesis}

Horizontal alveolar ridge augmentation through distraction osteogenesis demands for extreme preciseness in technique and the design of distractors. The amount of alveolar ridge width increase reported with distraction osteogenesis is $2.5 \mathrm{~mm}$ to $7 \mathrm{~mm}$. [84]- [89] Horizontal alveolar distraction can be conducted via simple bone screws to meticulously designed distractors. All devices have a distraction rod in common which is fixed in the cortical lingual/ palatal bone plate and allows for the buccal/ labial movement of the transfer segment. [84] Nevertheless, the intricacy of device positioning as well as the difficulty of performing an osteotomy in a narrow alveolar ridge have greatly restricted the indication of horizontal alveolar distraction osteogenesis. [85] Therefore, in many cases with horizontal alveolar deficiency, bone grafting techniques become advantageous over distraction osteogenesis. 

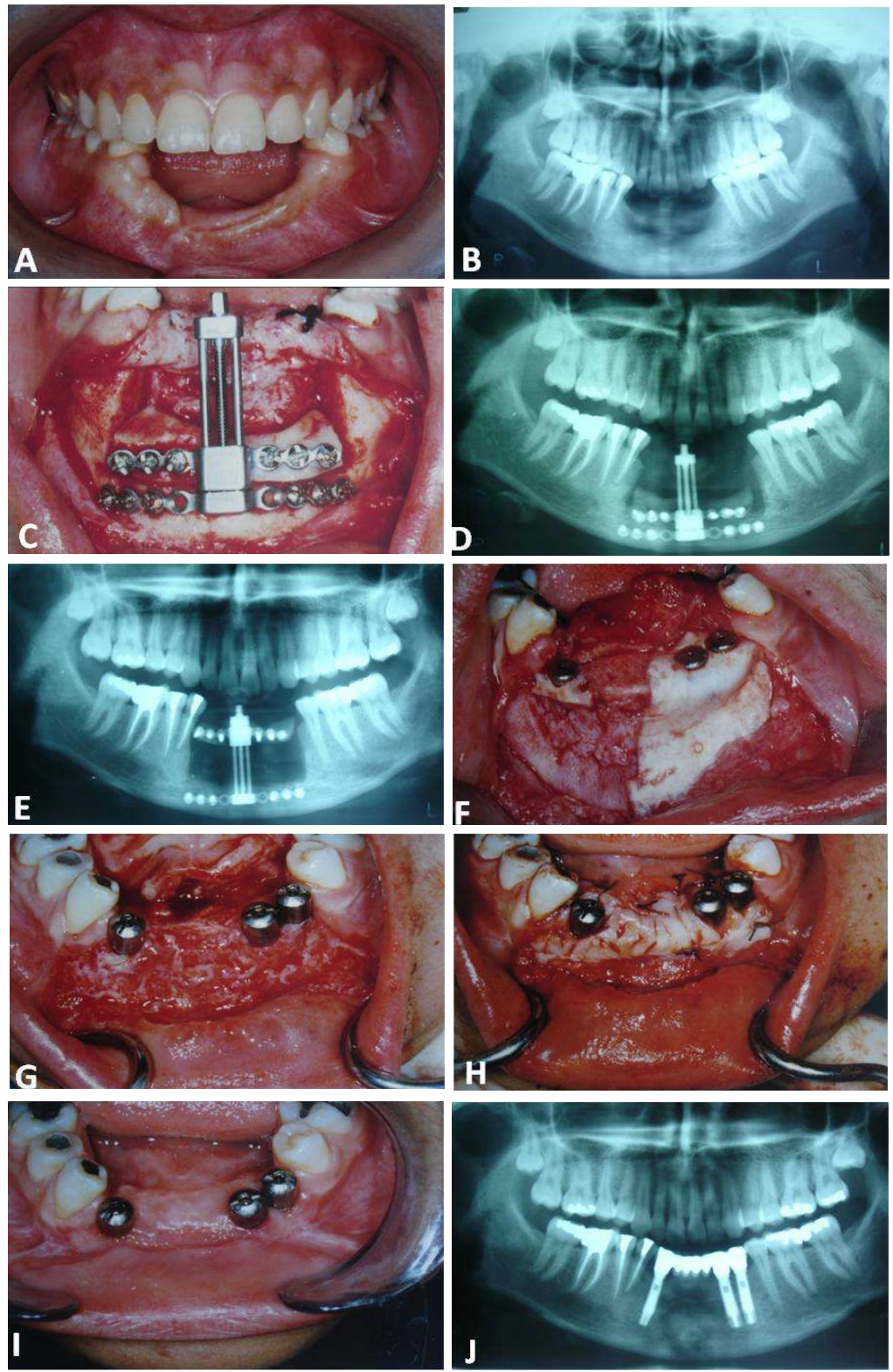

Figure 12. A. Large bone defect in the anterior mandible due to resection of a central giant cell granuloma. Intraoral view. B. Large anterior mandibular defect. Panoramic view. C. Intraoral distractor was placed. D. Panoramic view. E. Post-distraction panoramic view $18 \mathrm{~mm}$ augmentation was achieved. F. As a result of insufficient ridge width, a guided bone regeneration was done with implant placement. G. Second stage implant surgery, 3 months later. H. Inadequate keratinized tissue was compensated by a connective tissue graft harvested from the palate. I. Two months following the second stage implant surgery. J. Fixed implant-supported prosthesis was placed. Panoramic view. 


\section{Acknowledgements}

The authors wish to express their sincere gratitude to Dr. Ladan Eslamian; Professor of Department of Orthodontics, School of Dentistry, Shahid Beheshti university of Medical Sciences who took the responsiblity for orthodontic management of one of our patients, presented as case 9 .

\section{Author details}

Hossein Behnia ${ }^{1 *}$, Azita Tehranchi ${ }^{2}$ and Golnaz Morad ${ }^{3}$

1 Department of Oral and Maxillofacial Surgery, School of Dentistry, Shahid Beheshti University of Medical Sciences, Tehran, Iran

2 Department of Orthodontics, School of Dentistry, Shahid Beheshti University of Medical Sciences, Tehran, Iran

3 Dental Research Center, Shahid Beheshti University of Medical Sciences, Tehran, Iran

\section{References}

[1] Swennen G, Schliephake H, Dempf R, Schierle H, Malevez C. Craniofacial distraction osteogenesis: a review of the literature: Part 1: clinical studies. Int J Oral Maxillofac Surg 2001;30(2):89-103.

[2] Codivilla A. The classic: On the means of lengthening, in the lower limbs, the muscles and tissues which are shortened through deformity. 1905. Clin Orthop Relat Res 2008;466(12):2903-9.

[3] Abbott LC, Saunders JB. The Operative Lengthening of the Tibia and Fibula: a Preliminary Report on the Further Development of the Principles and Technic. Ann Surg 1939;110(6):961-91.

[4] McCarthy JG, Schreiber J, Karp N, Thorne CH, Grayson BH. Lengthening the human mandible by gradual distraction. Plast Reconstr Surg 1992;89(1):1-8; discussion 9-10.

[5] Ai-Aql ZS, Alagl AS, Graves DT, Gerstenfeld LC, Einhorn TA. Molecular mechanisms controlling bone formation during fracture healing and distraction osteogenesis. J Dent Res 2008;87(2):107-18.

[6] Marsh DR, Li G. The biology of fracture healing: optimising outcome. Br Med Bull 1999;55(4):856-69. 
[7] Morgan EF, Gleason RE, Hayward LN, Leong PL, Palomares KT. Mechanotransduction and fracture repair. J Bone Joint Surg Am 2008;90 Suppl 1:25-30.

[8] Ilizarov GA. The tension-stress effect on the genesis and growth of tissues. Part I. The influence of stability of fixation and soft-tissue preservation. Clin Orthop Relat Res 1989(238):249-81.

[9] Fischgrund J, Paley D, Suter C. Variables affecting time to bone healing during limb lengthening. Clin Orthop Relat Res 1994(301):31-7.

[10] Aronson J. Experimental and clinical experience with distraction osteogenesis. Cleft Palate Craniofac J 1994;31(6):473-81; discussion 81-2.

[11] Aronson J, Shen X. Experimental healing of distraction osteogenesis comparing metaphyseal with diaphyseal sites. Clin Orthop Relat Res 1994(301):25-30.

[12] Moore C, Campbell PM, Dechow PC, Ellis ML, Buschang PH. Effects of latency on the quality and quantity of bone produced by dentoalveolar distraction osteogenesis. Am J Orthod Dentofacial Orthop;140(4):470-8.

[13] Aronson J, Good B, Stewart C, Harrison B, Harp J. Preliminary studies of mineralization during distraction osteogenesis. Clin Orthop Relat Res 1990(250):43-9.

[14] Vauhkonen M, Peltonen J, Karaharju E, Aalto K, Alitalo I. Collagen synthesis and mineralization in the early phase of distraction bone healing. Bone Miner 1990;10(3): 171-81.

[15] Ilizarov GA. The tension-stress effect on the genesis and growth of tissues: Part II. The influence of the rate and frequency of distraction. Clin Orthop Relat Res 1989(239):263-85.

[16] Merloz P. Bone regeneration and limb lengthening. Osteoporos Int;22(6):2033-6.

[17] Michieli S, Miotti B. Lengthening of mandibular body by gradual surgical-orthodontic distraction. J Oral Surg 1977;35(3):187-92.

[18] Snyder CC, Levine GA, Swanson HM, Browne EZ, Jr. Mandibular lengthening by gradual distraction. Preliminary report. Plast Reconstr Surg 1973;51(5):506-8.

[19] Altug-Atac AT, Grayson BH, McCarthy JG. Comparison of skeletal and soft-tissue changes following unilateral mandibular distraction osteogenesis. Plast Reconstr Surg 2008;121(5):1751-9.

[20] Ow A, Cheung LK. Skeletal stability and complications of bilateral sagittal split osteotomies and mandibular distraction osteogenesis: an evidence-based review. J Oral Maxillofac Surg 2009;67(11):2344-53.

[21] Schreuder WH, Jansma J, Bierman MW, Vissink A. Distraction osteogenesis versus bilateral sagittal split osteotomy for advancement of the retrognathic mandible: a review of the literature. Int J Oral Maxillofac Surg 2007;36(2):103-10. 
[22] Kaban LB, Moses MH, Mulliken JB. Surgical correction of hemifacial microsomia in the growing child. Plast Reconstr Surg 1988;82(1):9-19.

[23] Padwa BL, Mulliken JB, Maghen A, Kaban LB. Midfacial growth after costochondral graft construction of the mandibular ramus in hemifacial microsomia. J Oral Maxillofac Surg 1998;56(2):122-7; discussion 27-8.

[24] Pereira MA, Luiz de Freitas PH, da Rosa TF, Xavier CB. Understanding distraction osteogenesis on the maxillofacial complex: a literature review. J Oral Maxillofac Surg 2007;65(12):2518-23.

[25] Tae KC, Kang KH, Kim SC. Unilateral mandibular widening with distraction osteogenesis. Angle Orthod 2005;75(6):1053-60.

[26] McCarthy JG, Staffenberg DA, Wood RJ, Cutting CB, Grayson BH, Thorne CH. Introduction of an intraoral bone-lengthening device. Plast Reconstr Surg 1995;96(4): 978-81.

[27] Boccaccio A, Cozzani M, Pappalettere C. Analysis of the performance of different orthodontic devices for mandibular symphyseal distraction osteogenesis. Eur J Orthod; 33(2):113-20.

[28] Raoul G, Wojcik T, Ferri J. Outcome of mandibular symphyseal distraction osteogenesis with bone-borne devices. J Craniofac Surg 2009;20(2):488-93.

[29] Shetye PR, Warren SM, Brown D, Garfinkle JS, Grayson BH, McCarthy JG. Documentation of the incidents associated with mandibular distraction: introduction of a new stratification system. Plast Reconstr Surg 2009;123(2):627-34.

[30] Ow AT, Cheung LK. Meta-analysis of mandibular distraction osteogenesis: clinical applications and functional outcomes. Plast Reconstr Surg 2008;121(3):54e-69e.

[31] Kofod T, Norholt SE, Pedersen TK, Jensen J. Unilateral mandibular ramus elongation by intraoral distraction osteogenesis. J Craniofac Surg 2005;16(2):247-54.

[32] Shetye PR, Grayson BH, Mackool RJ, McCarthy JG. Long-term stability and growth following unilateral mandibular distraction in growing children with craniofacial microsomia. Plast Reconstr Surg 2006;118(4):985-95.

[33] Tehranchi A, Behnia H. Facial symmetry after distraction osteogenesis and orthodontic therapy. Am J Orthod Dentofacial Orthop 2001;120(2):149-53.

[34] Tehranchi A, Behnia H. Treatment of mandibular asymmetry by distraction osteogenesis and orthodontics: a report of four cases. Angle Orthod 2000;70(2):165-74.

[35] Wang X, Wang XX, Liang C, Yi B, Lin Y, Li ZL. Distraction osteogenesis in correction of micrognathia accompanying obstructive sleep apnea syndrome. Plast Reconstr Surg 2003;112(6):1549-57; discussion 58-9. 
[36] Havlik RJ, Bartlett SP. Mandibular distraction lengthening in the severely hypoplastic mandible: a problematic case with tongue aplasia. J Craniofac Surg 1994;5(5): 305-10; discussion 11-2.

[37] Moore MH, Guzman-Stein G, Proudman TW, Abbott AH, Netherway DJ, David DJ. Mandibular lengthening by distraction for airway obstruction in Treacher-Collins syndrome. J Craniofac Surg 1994;5(1):22-5.

[38] PR.

[39] Feiyun P, Wei L, Jun C, Xin X, Zhuojin S, Fengguo Y. Simultaneous correction of bilateral temporomandibular joint ankylosis with mandibular micrognathia using internal distraction osteogenesis and 3-dimensional craniomaxillofacial models. J Oral Maxillofac Surg;68(3):571-7.

[40] Senders CW, Kolstad CK, Tollefson TT, Sykes JM. Mandibular distraction osteogenesis used to treat upper airway obstruction. Arch Facial Plast Surg;12(1):11-5.

[41] Scott AR, Tibesar RJ, Lander TA, Sampson DE, Sidman JD. Mandibular distraction osteogenesis in infants younger than 3 months. Arch Facial Plast Surg;13(3):173-9.

[42] Shen W, Jie C, Chen J, Zou J, Ji Y. Mandibular distraction osteogenesis to relieve Pierre Robin severe airway obstruction in neonates: indication and operation. J Craniofac Surg 2009;20 Suppl 2:1812-6.

[43] Kita H, Kochi S, Yamada A, Imai Y, Konno N, Saitou C, et al. Mandibular widening by distraction osteogenesis in the treatment of a constricted mandible and telescopic bite. Cleft Palate Craniofac J 2004;41(6):664-73.

[44] Conley R, Legan H. Mandibular symphyseal distraction osteogenesis: diagnosis and treatment planning considerations. Angle Orthod 2003;73(1):3-11.

[45] Sukurica Y, Gurel HG, Mutlu N. Six year follow-up of a patient treated with mandibular symphyseal distraction osteogenesis. J Craniomaxillofac Surg;38(1):26-31.

[46] Gurrereo.

[47] Chung YW, Tae KC. Dental stability and radiographic healing patterns after mandibular symphysis widening with distraction osteogenesis. Eur J Orthod 2007;29(3): 256-62.

[48] Rachmiel A, Jackson IT, Potparic Z, Laufer D. Midface advancement in sheep by gradual distraction: a 1-year follow-up study. J Oral Maxillofac Surg 1995;53(5):525-9.

[49] Staffenberg DA, Wood RJ, McCarthy JG, Grayson BH, Glasberg SB. Midface distraction advancement in the canine without osteotomies. Ann Plast Surg 1995;34(5): $512-7$. 
[50] Cohen SR, Burstein FD, Stewart MB, Rathburn MA. Maxillary-midface distraction in children with cleft lip and palate: a preliminary report. Plast Reconstr Surg 1997;99(5):1421-8.

[51] Mommaerts MY. Transpalatal distraction as a method of maxillary expansion. Br J Oral Maxillofac Surg 1999;37(4):268-72.

[52] Cheung LK, Chua HD. A meta-analysis of cleft maxillary osteotomy and distraction osteogenesis. Int J Oral Maxillofac Surg 2006;35(1):14-24.

[53] Meling TR, Hogevold HE, Due-Tonnessen BJ, Skjelbred P. Midface distraction osteogenesis: internal vs. external devices. Int J Oral Maxillofac Surg;40(2):139-45.

[54] Picard A, Diner PA, Galliani E, Tomat C, Vazquez MP, Carls FP. Five years experience with a new intraoral maxillary distraction device (RID). Br J Oral Maxillofac Surg;49(7):546-51.

[55] Aksu M, Saglam-Aydinatay B, Akcan CA, El H, Taner T, Kocadereli I, et al. Skeletal and dental stability after maxillary distraction with a rigid external device in adult cleft lip and palate patients. J Oral Maxillofac Surg;68(2):254-9.

[56] Mitsukawa N, Satoh K, Morishita T. Le Fort I distraction using internal devices for maxillary hypoplasia in patients with cleft lip, palate, and alveolus: complications and their prevention and management. J Craniofac Surg;21(5):1428-30.

[57] Richardson S, Agni NA, Selvaraj D. Anterior maxillary distraction using a toothborne device for hypoplastic cleft maxillas-a pilot study. J Oral Maxillofac Surg; 69(12):e542-8.

[58] Marchac A, Arnaud E. Cranium and midface distraction osteogenesis: current practices, controversies, and future applications. J Craniofac Surg;23(1):235-8.

[59] Kuroda S, Watanabe K, Ishimoto K, Nakanishi H, Moriyama K, Tanaka E. Long-term stability of LeFort III distraction osteogenesis with a rigid external distraction device in a patient with Crouzon syndrome. Am J Orthod Dentofacial Orthop;140(4):550-61.

[60] Ko EW, Chen PK, Tai IC, Huang CS. Fronto-facial monobloc distraction in syndromic craniosynostosis. Three-dimensional evaluation of treatment outcome and facial growth. Int J Oral Maxillofac Surg;41(1):20-7.

[61] Fearon JA. Halo distraction of the Le Fort III in syndromic craniosynostosis: a longterm assessment. Plast Reconstr Surg 2005;115(6):1524-36.

[62] Meazzini MC, Allevia F, Mazzoleni F, Ferrari L, Pagnoni M, Iannetti G, et al. Longterm follow-up of syndromic craniosynostosis after Le Fort III halo distraction: a cephalometric and CT evaluation. J Plast Reconstr Aesthet Surg;65(4):464-72.

[63] Cortese A, Savastano M, Savastano G, Claudio PP. One-step transversal palatal distraction and maxillary repositioning: technical considerations, advantages, and longterm stability. J Craniofac Surg;22(5):1714-9. 
[64] Seeberger R, Kater W, Schulte-Geers M, Davids R, Freier K, Thiele O. Changes after surgically-assisted maxillary expansion (SARME) to the dentoalveolar, palatal and nasal structures by using tooth-borne distraction devices. Br J Oral Maxillofac Surg; 49(5):381-5.

[65] Verlinden CR, Gooris PG, Becking AG. Complications in transpalatal distraction osteogenesis: a retrospective clinical study. J Oral Maxillofac Surg;69(3):899-905.

[66] Verstraaten J, Kuijpers-Jagtman AM, Mommaerts MY, Berge SJ, Nada RM, Schols JG. A systematic review of the effects of bone-borne surgical assisted rapid maxillary expansion. J Craniomaxillofac Surg;38(3):166-74.

[67] Block MS, Chang A, Crawford C. Mandibular alveolar ridge augmentation in the dog using distraction osteogenesis. J Oral Maxillofac Surg 1996;54(3):309-14.

[68] Chin M, Toth BA. Distraction osteogenesis in maxillofacial surgery using internal devices: review of five cases. J Oral Maxillofac Surg 1996;54(1):45-53; discussion 54.

[69] Turker N, Basa S, Vural G. Evaluation of osseous regeneration in alveolar distraction osteogenesis with histological and radiological aspects. J Oral Maxillofac Surg 2007;65(4):608-14.

[70] Perry M, Hodges N, Hallmon DW, Rees T, Opperman LA. Distraction osteogenesis versus autogenous onlay grafting. Part I: outcome of implant integration. Int J Oral Maxillofac Implants 2005;20(5):695-702.

[71] Uckan S, Oguz Y, Bayram B. Comparison of intraosseous and extraosseous alveolar distraction osteogenesis. J Oral Maxillofac Surg 2007;65(4):671-4.

[72] Bianchi A, Felice P, Lizio G, Marchetti C. Alveolar distraction osteogenesis versus inlay bone grafting in posterior mandibular atrophy: a prospective study. Oral Surg Oral Med Oral Pathol Oral Radiol Endod 2008;105(3):282-92.

[73] Jensen OT, Block M. Alveolar modification by distraction osteogenesis. Atlas Oral Maxillofac Surg Clin North Am 2008;16(2):185-214.

[74] Nocini PF, Albanese M, Buttura da Prato E, D'Agostino A. Vertical distraction osteogenesis of the mandible applied to an iliac crest graft: report of a case. Clin Oral Implants Res 2004;15(3):366-70.

[75] Dinse WE, Burnett RR. Anterior maxillary restoration using distraction osteogenesis and implants: a clinical report. J Prosthet Dent 2008;100(4):250-3.

[76] Gozneli R, Ozkan Y, Akalin ZF, Ozkan Y. Rehabilitation of maxillary anterior esthetics by alveolar distraction osteogenesis with immediate implant placement: a case report. Implant Dent;19(6):468-76.

[77] Lee HJ, Ahn MR, Sohn DS. Piezoelectric distraction osteogenesis in the atrophic maxillary anterior area: a case report. Implant Dent 2007;16(3):227-34. 
[78] Penarrocha-Diago M, Gomez-Adrian MD, Garcia-Garcia A, Camacho-Alonso F, Rambla-Ferrer J. Vertical mandibular alveolar bone distraction and dental implant placement: a case report. J Oral Implantol 2006;32(3):137-41.

[79] Rachmiel A, Gutmacher Z, Blumenfeld I, Peled M, Laufer D. [Vertical alveolar ridge augmentation using distraction osteogenesis]. Refuat Hapeh Vehashinayim 2001;18(1):64-9, 78.

[80] Raghoebar GM, Liem RS, Vissink A. Vertical distraction of the severely resorbed edentulous mandible: a clinical, histological and electron microscopic study of 10 treated cases. Clin Oral Implants Res 2002;13(5):558-65.

[81] Yalcin S, Ordulu M, Emes Y, Gur H, Aktas I, Caniklioglu C. Alveolar distraction osteogenesis before placement of dental implants. Implant Dent 2006;15(1):48-52.

[82] Perdijk FB, Meijer GJ, Strijen PJ, Koole R. Complications in alveolar distraction osteogenesis of the atrophic mandible. Int J Oral Maxillofac Surg 2007;36(10):916-21.

[83] McAllister BS, Gaffaney TE. Distraction osteogenesis for vertical bone augmentation prior to oral implant reconstruction. Periodontol 2000 2003;33:54-66.

[84] Aikawa T, Iida S, Senoo H, Hori K, Namikawa M, Okura M, et al. Widening a narrow posterior mandibular alveolus following extirpation of a large cyst: a case treated with a titanium mesh-plate type distractor. Oral Surg Oral Med Oral Pathol Oral Radiol Endod 2008;106(5):e1-7.

[85] Bulut E, Muglali M, Celebi N, Bekcioglu B. Horizontal alveolar distraction of the mandibular canine regions for implant placement. J Craniofac Surg;21(3):830-2.

[86] Garcia-Garcia A, Somoza-Martin M, Gandara-Vila P, Saulacic N, Gandara-Rey JM. Horizontal alveolar distraction: a surgical technique with the transport segment pedicled to the mucoperiosteum. J Oral Maxillofac Surg 2004;62(11):1408-12.

[87] Laster Z, Rachmiel A, Jensen OT. Alveolar width distraction osteogenesis for early implant placement. J Oral Maxillofac Surg 2005;63(12):1724-30.

[88] Oda T, Suzuki H, Yokota M, Ueda M. Horizontal alveolar distraction of the narrow maxillary ridge for implant placement. J Oral Maxillofac Surg 2004;62(12):1530-4.

[89] Watzak G, Zechner W, Tepper G, Vasak C, Busenlechner D, Bernhart T. Clinical study of horizontal alveolar distraction with modified micro bone screws and subsequent implant placement. Clin Oral Implants Res 2006;17(6):723-9. 